\title{
Percepciones de confort en una edificación histórica de uso educativo, caso de estudio: Facultad Ciencias Económicas - Universidad Central del Ecuador
}

\section{Perceptions of comfort in a historic building for educational use case study: Faculty of Economics - Universidad Central del Ecuador}

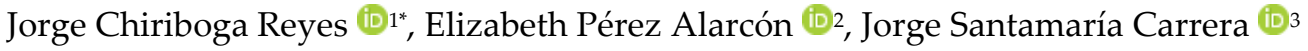 \\ ${ }^{1}$ Facultad de Arquitectura y Urbanismo, Universidad Central del Ecuador, Quito, Ecuador, 170136 \\ ${ }^{2}$ Facultad de Filosofía, Letras y Ciencias de la Educación, Universidad Central del Ecuador, Quito-Ecuador, 170136; \\ eyperez@uce.edu.ec \\ ${ }^{3}$ Facultad de Ingeniería y Ciencias Aplicadas, Universidad Central del Ecuador, Quito-Ecuador, 170136; jsantamaria@uce.edu.ec
}

Citación: Chiriboga, J., Pérez, E. \& Santamaría, J., (2021). Percepciones de confort en una edificación histórica de uso educativo, Caso de estudio: Facultad Ciencias Económicas Universidad Central. Novasinergia. 4(1) 74-90.

https://doi.org/10.37135/ns.01.07.04

Recibido: 12 marzo 2021

Aceptado: 20 mayo 2021

Publicado: 01 junio 2021

Novasinergia

ISSN: 2631-2654

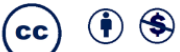

Copyright: 2021 derechos otorgados por los autores a Novasinergia.

Este es un artículo de acceso abierto distribuido bajo los términos y condiciones de una licencia de Creative Commons Attribution (CC BY NC). (http://creativecommons.org/licenses/b y/4.0/).
*Correspondencia: jvchiriboga@uce.edu.ec

Resumen: En el artículo se analizan las percepciones de confort de los estudiantes que asisten al Bloque "A", de la Facultad de Ciencias Económicas - Universidad Central del Ecuador (UCE). El problema radica en conocer si esta edificación, cumple con las demandas actuales de confort ambiental, psicológico, funcional y eficiencia energética. Es un estudio descriptivo cuyo objetivo fue evaluar las percepciones de las variables citadas en el problema. Se aplicó una encuesta tipo Likert a una muestra de estudiantes. El nivel de confianza y margen de error fueron 95 \% y 7 \% respectivamente. Se emplearon el Índice de Importancia Relativa y $\mathrm{Chi}^{2}$ para el procesamiento de la información. Se determinó que las condiciones ambientales y psicológicas son más confortables en el ambiente exterior, pero se prefiere el ambiente interior para realizar las actividades académicas. Factores que inciden en el confort en orden decreciente son: actividades académicas al interior, iluminación natural exterior, espacios de encuentro y diálogo. Las variables de incomodidad fueron ruidos y olores desagradables. Respecto del espacio físico, la edificación cumple con el aforo normado. Las percepciones de los estudiantes son favorables respecto de las variables estudiadas en la edificación. En conclusión, la edificación ofrece confort en los componentes analizados.

Palabras clave: Ambientes, confort ambiental, confort psicológico, eficiencia energética, habitabilidad, percepción del confort.

\begin{abstract}
The article analyzes the comfort perceptions of the students who attend Building " $A$ " of the Faculty of Economic Sciences - Central University of Ecuador. The problem lies in knowing if this building meets the current demands of environmental, psychological, functional comfort, and energy efficiency. It is a descriptive study whose objective was to evaluate the perceptions of the variables mentioned in the problem. A Likert-type survey was applied to a sample of students. The level of confidence and margin of error were $95 \%$ and $7 \%$, respectively. The Relative Importance Index and Chi ${ }^{2}$ were used for information processing. It was determined that the environmental and psychological conditions are more comfortable in the outdoor environment. However, the indoor environment is preferred to carry out academic activities. Factors that affect comfort in decreasing order are indoor academic activities, outdoor natural lighting, meeting and dialogue spaces. The discomfort variables were unpleasant noises and odors. Regarding the physical space, the building complies with the regulated capacity. The students' perceptions are favorable regarding the variables studied in the building. In conclusion, the building offers comfort in the components analyzed.
\end{abstract}

Keywords: Comfort perception, environments, energy efficiency, environmental comfort, habitability, psychological comfort. 


\section{Introducción}

Temas relevantes como el confort ambiental, la eficiencia energética, la habitabilidad y su incidencia en el patrimonio construido, son temas que demandan mayor dedicación y tiempo de investigación. Paralelamente, otros problemas como la destrucción directa o indirecta del ambiente natural, el crecimiento exponencial de desechos, la contaminación en general, el efecto del calentamiento del planeta, plantean una gran problemática que debe ser abordada para idear soluciones emergentes y urgentes.

En el Ecuador en la última década se han hecho esfuerzos por contar con leyes, normativas y reglamentos que favorezcan la sustentabilidad y eficiencia energética. En este sentido, se consideran algunas normativas relevantes como las citadas por el Instituto Nacional de Eficiencia Energética (INER) con la publicación Estrategias para mejorar las condiciones de habitabilidad y el consumo de energía en viviendas de Godoy et al., (2017) donde se hace un primer acercamiento hacia la definición climática habitacional del país.

El Instituto Ecuatoriano de Normalización (INEN) publica la Norma Técnica Ecuatoriana NTE INEN 2506:2009 sobre la Eficiencia Energética en Edificaciones (INEN, 2009). El Ministerio de Desarrollo Urbano y Vivienda (2018) propone la Norma Ecuatoriana de la Construcción NEC 11 la cual en su capítulo 13 trata la Eficiencia Energética en la Construcción en Ecuador y en el capítulo 14 las Energías Renovables. La citada norma establece en estos capítulos lineamientos, normativas y criterios urbanísticos, arquitectónicos y constructivos, tipos de confort y aspectos energéticos con criterios de sostenibilidad. Además, se dispone de la ley orgánica de Eficiencia Energética (Asamblea Nacional del Ecuador, 2019).

Sobre la base de lo expuesto, con relación a los documentos referidos que constituyen leyes, normativas y reglamentos, en la presente investigación se evalúa si las edificaciones con valor histórico, arquitectónico y social reúnen las condiciones de confort necesarias en cuanto a sensaciones perceptuales de confort térmico y eficiencia energética, niveles de confort dados desde la percepción de los elementos psicológicos, ambientales y de la eficiencia energética.

Para ello se toma como caso de estudio a una de las edificaciones simbólicas y emblemáticas de la UCE, creadas por el connotado arquitecto uruguayo Gilberto Gatto Sobral en 1957 (Peralta \& Moya, 2007). Corresponde al edificio de la Facultad de Ciencias Económicas que formó parte de la primera etapa del proyecto general de la ciudadela universitaria en 1947, que se diseñó como un conjunto arquitectónico. Según lo citado por Villagómez \& Bonilla (2017), este edificio forma parte de un todo constructivo y refleja la fusión de la arquitectura con el contexto. Este proyecto obtuvo el premio al Ornato Municipal de Quito en 1959 (Peralta \& Moya, 2007). La edificación fue diseñada y construida para uso exclusivamente educativo desde hace más de seis décadas. Es preciso destacar que este inmueble no ha sido sometido a remodelaciones o adaptaciones de su uso original ni modificaciones a sus tipologías arquitectónicas.

El edificio Bloque A se organiza de forma longitudinal en sentido norte-sur y está definido por una secuencia de tres volúmenes semi circulares que para efectos de este trabajo se puede denominar sur, centro y norte, que conforman un solo cuerpo. El conjunto está organizado espacialmente en tres niveles en los cuales se ubican los diferentes ambientes que lo componen. En el cuerpo sur o bloque de aulas se observa claramente una disposición y organización modular en hilera de las aulas en cada planta, las circulaciones y la estructura. Es preciso anotar que el edificio maneja un diálogo armonioso de lo construido con los espacios abiertos exteriores, caminerías, circulaciones, jardines y áreas verdes que en conjunto expresan armonía y calidad espacial, formal y estética. 
Estudios de temáticas relacionadas o complementarias se vienen desarrollado en universidades ecuatorianas como la Universidad de Cuenca (Galindo, 2017), Universidad Politécnica Salesiana de Cuenca (Arévalo \& Arias, 2017), Universidad Técnica de Ambato, (Jiménez, 2017), Universidad Católica de Quito (Riofrío, 2019), UCE (Baldeón, 2015). En estas instituciones de educación superior se abordan importantes investigaciones sobre temas ambientales, de sustentabilidad, arquitectura bioclimática, confort térmico y eficiencia energética sea en calidad de trabajos de titulación a nivel de posgrado o como trabajos de titulación de pregrado como se indica a continuación.

Es preciso recalcar el aporte del estudio titulado Análisis del Gradiente Térmico para alcanzar condiciones de confort humano en una de las aulas de la Universidad Politécnica Salesiana de Cuenca, que trata sobre la incidencia de la temperatura en la misma que provoca distorsiones del margen de confort térmico debido a las condiciones adversas que presentan los materiales con los cuales está construida. Sobre todo, el material del recubrimiento de cubierta que es metálico el cual genera aumento de temperatura al interior del aula por la radiación solar, la falta de una adecuada ventilación y la carencia de un estudio de asoleamiento que permita una mejor orientación del aula, así también el tamaño de las ventanas que produce reflejo hacia el pizarrón aspectos que en su conjunto afectan el proceso cognitivo de enseñanza-aprendizaje (Arévalo \& Arias, 2017). Lo citado permite evidenciar que un espacio educativo desde su concepción debe cumplir con lineamientos y normativas de diseño que garanticen el confort de sus ocupantes.

El interés por estos temas también se ve reflejado en trabajos realizados por investigadores individuales y grupos de investigación con la publicación de artículos científicos sobre temáticas vinculadas en países Hispanoamericanos como Ecuador, Argentina, España, México, entre otros. Sin embargo, en lo relacionado con espacios donde se desarrollan actividades educativas en estructuras edificadas antiguas, las fuentes de consulta son limitadas en el país.

Del mismo modo, trabajos desarrollados por centros de educación superior como la Universidad de Tucumán (Cisterna et al., 2015), la Universidad Nacional del Nordeste en Argentina (Alías et al., 2011), Universidad Politécnica de Valencia (Ortíz, 2016), Universidad Metropolitana México (Fuentes, 2002), entre otras, que si bien tratan temas ambientales, de sustentabilidad, arquitectura bioclimática, confort térmico y eficiencia energética, no se enfocan de manera directa en la relación entre el confort ambiental y la habitabilidad en edificaciones históricas. Como referencia se puede mencionar que en la Universidad Nacional de Cajamarca se realizó una investigación que no profundiza en temas ambientales, pero pone de manifiesto que en la actualidad existen edificaciones coloniales que tienen uso educativo pero su estado de conservación constituye un riesgo para sus ocupantes (Gómez, 2018).

De igual manera, en la Universidad Politécnica de Cataluña Barcelonatech se registra una investigación que determina el vínculo entre las aulas escolares y la enseñanza, lo cual a su vez tiene relación con la teoría del diseño arquitectónico y sus efectos en los procesos pedagógicos. Todo este trabajo está fortalecido con el análisis de los movimientos educativos a fin de ofrecer soluciones arquitectónicas escolares (Cardellino, Vargas, \& Araneda, 2017). Este trabajo, si bien no establece una relación del uso educativo con una edificación histórica, permite dar soporte al interés que existe por conocer cómo la arquitectura influye en el proceso de enseñanza aprendizaje.

Las perspectivas ambiental y psicológica son complementarias en la determinación del confort de una edificación. Concierne a lo psicológico lo manifestado por De Garrido (2013), quien enfatiza que las edificaciones deben propender al bienestar de sus ocupantes. Lo cual es de suma importancia si se considera lo expuesto por Vásquez (2017), quien afirma que la confortabilidad está unida de manera directa con la productividad de los espacios. La sensación de protección y seguridad es otro 
aspecto psicológico que debe tratar de ser satisfecho. Por tal razón los espacios habitables deben proporcionar la mayor seguridad posible, en virtud que la mayor parte del tiempo de la vida el ser humano permanece al interior de las edificaciones (De Garrido, 2013).

El nivel de autosuficiencia manifestado a través de la satisfacción de las necesidades básicas de agua y energía es otro de los puntales de índole psicológico dado que su insatisfacción genera estrés en las personas. Si bien el cuerpo del ser humano tiene una buena capacidad de autorregulación térmica, sin embargo, para lograr comodidad o confort requiere de ambientes que no sean demasiado fríos ni calientes ya que el esfuerzo biológico de adaptación a dichas condiciones disminuye la energía del ser humano (Olgyay, 2019). Según el citado autor, el clima puede afectar al ser humano haciendo que entre en tensión, dolor, enfermedad y muerte, más, si es favorable, puede incidir en la productividad, la salud, la energía mental y física, donde el ser humano alcanza su máxima eficiencia.

Estos parámetros a ser estudiados forman parte de la arquitectura bioclimática y del confort físico de los espacios, sus condiciones físicas mínimas de habitabilidad que en el presente caso se aplicará a un uso educativo con el análisis de un grupo de aulas del Bloque A de la Facultad, además investigar como este confort se relaciona con la habitabilidad educativa.

En concordancia con los párrafos previamente citados, el presente trabajo se traduce en un aporte al conocimiento sobre las percepciones del confort de las condiciones ambientales, psicológicas, funcionales y eficiencia energética de una edificación de un centro educativo de educación superior. El aporte se sustenta en el estudio de las percepciones de confort y cómo éste incide en la habitabilidad de los espacios de la edificación. La condición sensorial y perceptual de los individuos es necesario evaluarla en este caso por tratarse de una edificación construida hace más de seis décadas, que, si bien fue diseñada para fines educativos, las demandas de confort actuales de los estudiantes pudiesen haber variado.

El objetivo de esta investigación es evaluar las sensaciones perceptuales de confort ambiental, psicológico, eficiencia energética y la calidad funcional de los espacios que los estudiantes experimentan durante su permanencia en las aulas de clase en horario diurno. La pregunta de investigación se enfoca en conocer ¿Cuál es la percepción sensorial y psicológica que los estudiantes tienen respecto de los parámetros de confort ambiental, psicológico, energético, funcional y condiciones de habitabilidad actuales en el Bloque A de la Facultad de Ciencias Económicas de la UCE?, ya que al tratarse de una edificación con valoración histórica y arquitectónica construida para uso educativo en la década de los 50's, en la actualidad podría no reunir las condiciones de confort. La respuesta a esta pregunta se constituye en una base objetiva de conocimiento para la elaboración de una propuesta de mejoramiento del confort y optimización de las actividades académicas. Además, permite generar otras líneas de investigación paralelas como el profundizar estudios sobre el estado de conservación. En la hipótesis formulada se afirma que el edificio denominado Bloque A de la Facultad de Ciencias Económicas responde a las demandas y necesidades de parámetros de confort ambiental, consumo energético y condiciones de habitabilidad necesarias al año 2020.

\section{Metodología}

\subsection{Diseño del estudio}

En la figura 1, se ilustra de manera secuencial las actividades desarrolladas por etapas en este estudio. La etapa 1, comprende el diseño del estudio, la etapa 2 se refiere a la selección de la muestra, 
en la etapa 3 se realiza la recolección de los datos y las pruebas estadísticas, la etapa 4 corresponde al análisis de datos, la etapa 5 muestra los resultados y conclusiones.

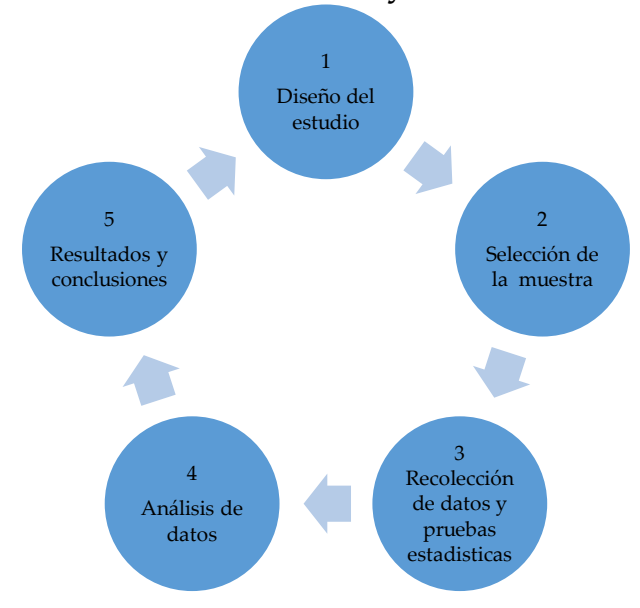

Figura 1: Etapas de la Metodología

La investigación se fundamenta en el confort, que según afirma Fuentes (2002) consiste en un estado de percepción ambiental momentáneo y en el análisis de los efectos que producen el confort térmico, visual, acústico y psicológico. La norma ASHRAE (2010) menciona al confort térmico como una sensación de la persona que expresa su satisfacción con el ambiente térmico. En el estudio se analiza el componente ambiental, psicológico, funcional y energético, a fin de estructurar una evaluación de las condiciones de confort y habitabilidad dentro de un grupo de aulas seleccionadas previamente a partir de la aplicación de una encuesta de satisfacción sobre las sensaciones y percepciones de los estudiantes durante el horario de clases.

El estudio es de naturaleza descriptiva, observacional de corte transversal y cuantitativa. Se llevó a cabo en el bloque A de la Facultad de Ciencias Económicas de la UCE en los meses de noviembre y diciembre de 2019. Consistió en la evaluación de las condiciones de confort ambiental y psicológico desde el punto de vista perceptual en estructuras edificadas con valoración histórica.

La evaluación comprende la elaboración y puesta en marcha de una encuesta de percepción y satisfacción de confort térmico, lumínico, acústico y psicológico conjuntamente con eficiencia energética. El instrumento se aplicó a los estudiantes que asisten a clases diariamente en horario matutino en las aulas del Bloque A de la Facultad de Ciencias Económicas de la UCE, proceso vinculado a la etapa 3 de la metodología.

\subsection{Población y Muestra}

La población estuvo constituida por 274 estudiantes que asistían a clases en horario matutino. Estuvieron distribuidos en 16 aulas (de las cuales 2 estuvieron destinadas a otros fines). La muestra se calculó según la fórmula citada en Jiménez, Logroño, Rodas, \& Yépez (1999), ecuación (1),

$$
n=\frac{P Q \times N}{\frac{(N-1) E^{2}}{K^{2}}+P Q}
$$

Donde $n$ es el tamaño de la muestra, $P Q$ la varianza media de la población (0.25), $N$ la población o universo, $E$ el error admisible y $K$ coeficiente de corrección del error (valor de 2).

El tamaño de la muestra calculado correspondió a 97 estudiantes, sin embargo, este número fue superado en 17 encuestados los cuales respondieron las encuestas con datos válidos, por tanto, se 
trabajó con 114 alumnos. El nivel de confianza de la muestra fue del 95 \% y el margen de error del 7 \% calculados sobre la base de Creative Researh System (1982).

Para la representatividad se aplicó el muestreo aleatorio simple en el que se tomaron estudiantes de las 14 aulas habilitadas. El grupo de estudiantes encuestados correspondió a los jóvenes que estudian en horario matutino y que en su mayoría eran de semestres iniciales en la facultad. Este grupo fue divido en dos subgrupos según la edad y de acuerdo al sexo ya que de acuerdo a lo expresado por Olgyay (2019), tanto el sexo como la edad cumplen una función preponderante en los requerimientos térmicos. Un subgrupo se conformó por los estudiantes menores a 20 años (61.4\%) y otro subgrupo con los jóvenes mayores a 20 años de edad (38.6\%). Respecto del sexo, la mayor cantidad lo conforman las mujeres (59.6 \%) seguido del grupo de varones (40.4\%).

\subsection{Mediciones y pruebas estadísticas}

El instrumento de investigación empleado fue una encuesta estructurada según la escala de Likert. Para la validez del instrumento se sometió a juicio de cinco expertos de la Facultad de Arquitectura y Urbanismo de la UCE. Su experticia se respalda en los años de docencia en el área arquitectura bioclimática, conservación del patrimonio y afines, así como en la publicación de documentos científicos. Además, la validez también se fundamentó en la aplicación de una prueba piloto a 15 estudiantes de la citada Facultad. Para la confiabilidad se aplicó el Alfa de Cronbach que dio como resultado un valor de 0.9 , mismo que indica que el instrumento es muy confiable. Una vez solventados los filtros de validez y confiabilidad del instrumento, se aplicó la encuesta mediante un cuestionario de 49 preguntas en línea con la aplicación Google Forms lográndose una tasa de respuesta del $42 \%$. Se llevó a cabo en noviembre y diciembre de 2019 antes de la crisis sanitaria.

Las preguntas estuvieron relacionadas con parámetros de confort ambiental como: sensaciones de temperatura, humedad, calidad del aire, niveles de ruido y también parámetros de confort arquitectónico relacionados con: características de la edificación, calidad de los espacios en cuanto a confort térmico, lumínico, acústico, eficiencia energética, energía renovable, iluminación-fuerza, agua potable y características de los ambientes interior y exterior.

\subsection{Análisis de datos}

La información obtenida se exportó a una base de datos elaborada en Microsoft Excel. Una vez depurada la base de datos se la exportó al programa estadístico Statistical Package for the Social Sciences (IBM SPSS versión 22. https://www.ibm.com/products/spss-statistics). En este se analizaron todas las preguntas de manera individual. Las 49 preguntas del cuestionario en SPSS se las agrupó en cinco secciones que corresponden a: 1) confort ambiente interior 2) confort psicológico interior 3) confort ambiente exterior 4) confort psicológico exterior y 5) patrimonio. El componente ambiental tiene como variables la temperatura, contaminación del aire, humedad del aire, ventilación e iluminación natural. Variables que se consideraron en respuesta a lo que manifiestan Narváez, Quezada, \& Villavicencio (2015), sobre los parámetros de confort que pueden ser ambientales como temperatura seca del aire, temperatura radiante, humedad relativa, calidad del aire, vientos, radiación solar y niveles de ruido, pues estos parámetros permiten valorar las condiciones de confort.

El componente psicológico comprende las variables: colores, seguridad, espacios para el encuentro y diálogo, gases u olores desagradables y ruidos perturbadores. Finalmente, el componente patrimonio tiene como variables la comodidad de los espacios para realizar actividades académicas, suficiencia de instalaciones eléctricas e instalaciones hidrosanitarias. 
En el análisis e interpretación de los resultados se empleó el Índice de Importancia Relativa (IIR) para determinar el orden de importancia de las variables dentro del confort en relación a los componentes ambiental, psicológico y patrimonial.

Las percepciones negativa e indiferente (rangos que reflejarían una carencia de afectividad) y positiva son catalogadas como una actitud de los estudiantes frente al objeto de análisis que en el presente caso son las percepciones sentidas frente a las distintas variables analizadas de la edificación de Facultad de Ciencias Económicas. Según Castejón (2006), las actitudes constituyen una mezcla de pensamientos y sentimientos, opiniones o percepciones, acerca de personas u objetos físicos, instituciones, etc.

Thurstone citado por Castejón (2006), señala que "una actitud es la intensidad del afecto positivo o negativo hacia un objeto psicológico" (p. 95) siendo este un "símbolo, persona, frase, eslogan o idea hacia el que las personas pueden diferir respecto al afecto positivo o negativo". En tal virtud, lo citado respalda y justifica la división y denominación de los rangos que reemplazan a las opciones iniciales dadas por Likert. En este sentido, el cambio de escala ayudó a resumir de mejor manera la variación de las percepciones. Finalmente, para la prueba de hipótesis se utilizó Chi cuadrado (Chi²).

\section{Resultados}

Los resultados giran en torno a la aplicación de la escala de Likert agrupada en dos categorías, una de percepción positiva y otra negativa, misma que a su vez abarca la posición de percepción indiferente, tal como se señaló en párrafos precedentes. Respecto a la determinación de la percepción del confort de los estudiantes de la Facultad de Ciencias Económicas, el resultado puede evidenciarse en la figura 2.

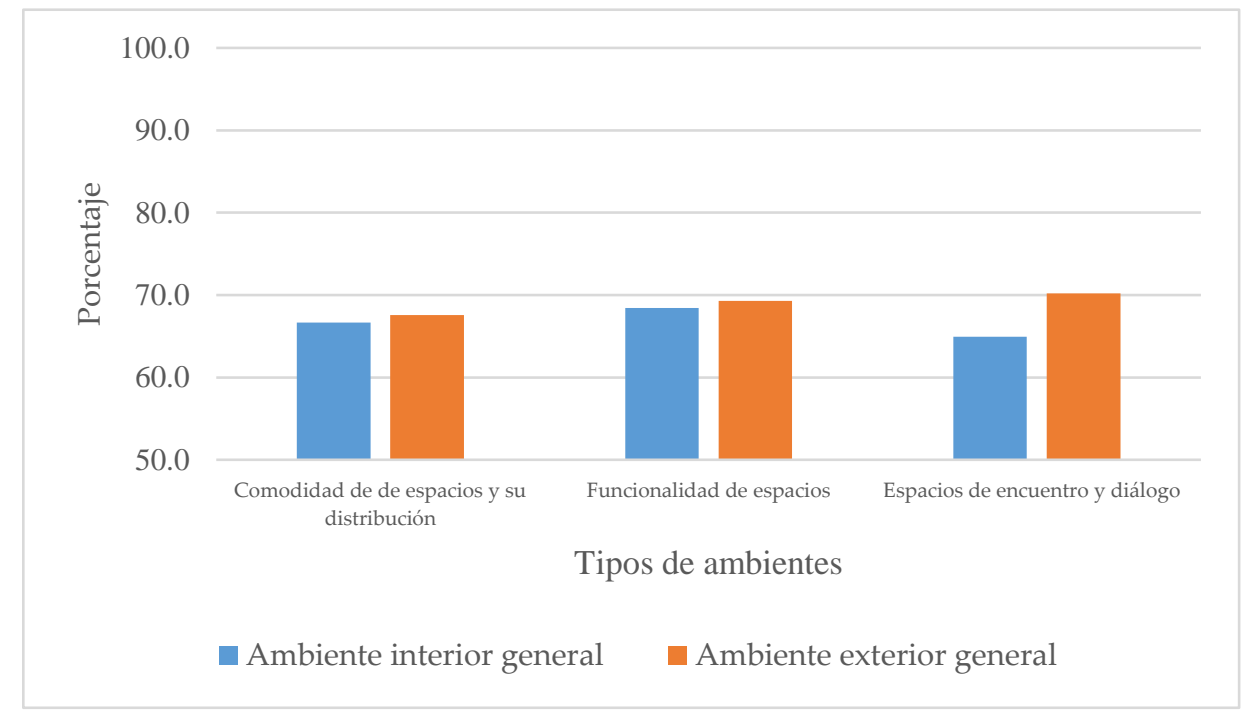

Figura 2: Percepción positiva en porcentajes de la comodidad de espacios, distribución y funcionalidad de los ambientes.

La figura 2 en el que se relacionan la comodidad de los espacios, distribución, funcionalidad y espacios de encuentro frente a los ambientes interior y exterior, muestra mayor porcentaje la percepción positiva del ambiente exterior $(67.5 \%, 69.3 \%$ y $70.2 \%)$ que la percepción positiva del ambiente interior $(66.7 \%, 68.4 \%$ y $64.9 \%)$ respectivamente en todas las variables previamente expuestas. 
Esto podría deberse a que las condiciones ambientales (temperatura, humedad, ventilación y luz natural) y las condiciones psicológicas (percepción de diseño exterior, colores y seguridad de los espacios) de los espacios exteriores ofrecen mayor comodidad para su permanencia.

En la figura 3 se visualiza que el confort ambiental del ambiente exterior es más valorado (78.8 \%) que el confort ambiental del ambiente interior (70.2\%), cuya diferencia en porcentaje representa el $8.6 \%$, lo que implicaría casi una décima parte más de preferencia hacia el ambiente exterior. Situación que podría deberse a que según De Garrido (2013) una condición primordial para sentir bienestar es que el ser humano se encuentre formando parte del medio natural.

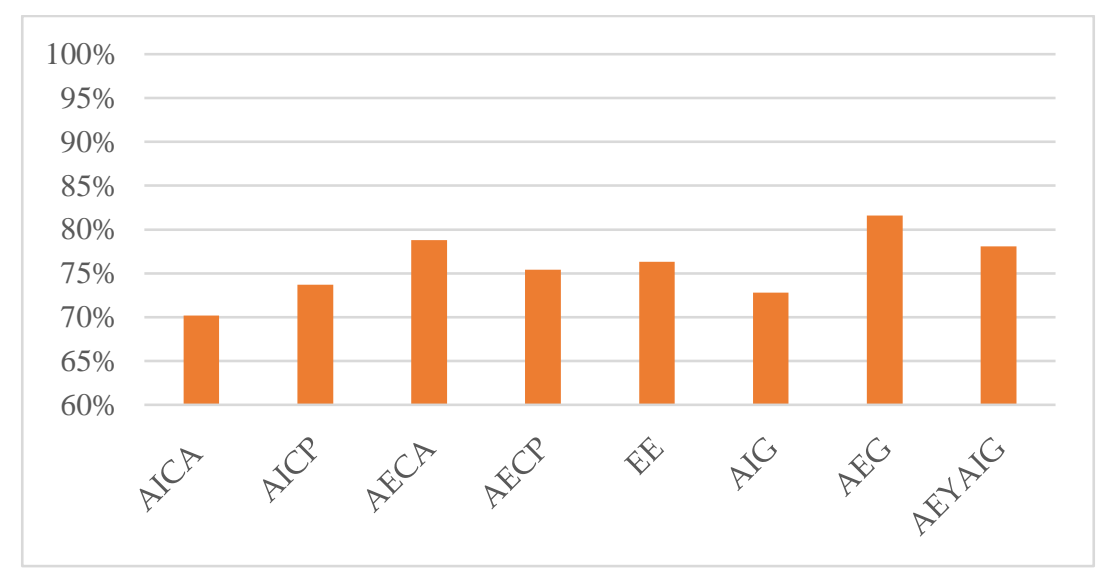

Figura 3: Ambientes, Eficiencia Energética, Confort y Patrimonio en porcentaje. AICA= Ambiente interior confort ambiental; $\mathrm{AICP}=\mathrm{Ambiente}$ interior confort psicológico; $\mathrm{AECA}=\mathrm{Ambiente}$ exterior confort ambiental; $\mathrm{AECP}=\mathrm{Ambiente}$ exterior confort psicológico; $\mathrm{EE}=$ Eficiencia energética; $\mathrm{AIG}=$ Ambiente interior general; $\mathrm{AEG}=\mathrm{Ambiente}$ exterior general; AEYAIG= Ambiente exterior y ambiente interior general.

El confort psicológico tanto en el ambiente interior (73.70\%) como en el ambiente exterior (75.40 \%) no difieren en gran medida la valoración. En los dos casos esta es positiva, sin embargo, existe una diferencia del $1.70 \%$ a favor del confort psicológico del ambiente exterior. Lo cual podría deberse a la influencia de los colores del exterior de la edificación dado que una parte es rosácea y otra parte blanca. Acorde a lo expresado por De Garrido (2013) el color rosa es muy indicado para estados de depresión leve y despeja la mente de los pensamientos negativos en tanto que el color blanco aporta vitalidad y fuerza.

De igual manera en el citado gráfico se evidencia que para realizar actividades académicas los estudiantes sienten mayor confort en el ambiente exterior general (81.6\%) que en el ambiente interior general $(72.8 \%)$. La diferencia en porcentaje es igual a $8.8 \%$ a favor del primero. Resultado que podría deberse a la confluencia de los factores antes estudiados es decir a la interacción que existe entre los factores ambientales y los psicológicos.

La variable de eficiencia energética y sus componentes analizados (agua, energía, iluminación natural) al cruzar con las variables de confort tanto interior como exterior, muestra un resultado altamente positivo de aceptación por parte de los estudiantes $(76.3 \%)$. Por lo que se puede inferir que el disponer de agua, energía iluminación, ofrece a los estudiantes sensación de bienestar lo cual se expresa con un valor alto de aceptación por parte de los mismos.

El 74\% del total de estudiantes encuestados responden de manera positiva a las condiciones de confort ambiental interior, en tanto que el $26 \%$ responde con criterios de indiferente o negativo. Del $74 \%$, el $57 \%$ corresponde al sexo femenino y el $43 \%$ al masculino. 
De los 114 estudiantes encuestados, que representan el $100 \%$ de la muestra, $40 \%$ (46) son hombres y $60 \%$ (68) son mujeres. Las proporciones alcanzadas en el estudio en relación al criterio de aceptación del ambiente interior, 43\% (36) son hombres 57 \% (48) son mujeres. Esto denota que se mantiene la tendencia porcentual mayor de las mujeres que de los hombres lo cual podría deberse a que en la población estudiantil en general hay más mujeres que hombres. Por lo tanto, otro de los criterios por los que las mujeres tienen mayor porcentaje puede estar vinculado con el mayor número de mujeres que forman parte de la muestra y que respondieron de manera positiva a la encuesta.

En la tabla 1, referente al confort ambiental interior relacionado a la edad por frecuencias, del total de la muestra se consideraron los 84 estudiantes que respondieron de manera positiva para el confort interior. Los grupos etarios fueron de 18-20, 21-25 y 26 años o más. Las frecuencias son 49, 32 y 3 respectivamente cuyos porcentajes responden al 58\%; 38\% y 4\% según el orden citado.

Tabla 1: Confort ambiental interior en relación a la edad por frecuencias

\begin{tabular}{ccc}
\hline $\begin{array}{c}\text { Variable: } \\
\text { Edad }\end{array}$ & $\begin{array}{c}\text { Frecuencia } \\
\text { (f) }\end{array}$ & $\begin{array}{c}\text { Porcentaje } \\
(\%)\end{array}$ \\
\hline $18-20$ & 49 & 58 \\
$21-25$ & 32 & 38 \\
$26+$ & 3 & 4 \\
Total & 84 & 100 \\
\hline
\end{tabular}

En el presente estudio no fue factible determinar la diferencia en la preferencia de los factores de confort ambiental interior de los grupos debido a que los estudiantes de la muestra no superan los 30 años de edad.

La mayoría de estudiantes que valoran de manera positiva al confort ambiental interior, corresponden al segmento de estudiantes más jóvenes. Esto debido a que la mayoría de la población estudiantil analizada corresponde a semestres iniciales, donde los estudiantes oscilan entre los 18 a 20 años.

\subsection{Espacio físico y normativa}

El análisis se enmarca en las percepciones y sensaciones respecto de las distintas variables energéticas, de confort ambiental y de confort físico relacionándolas con la calidad espacial de los espacios para mantener buenas condiciones de habitabilidad educativa y bienestar personal que favorezcan en los procesos de enseñanza aprendizaje. El levantamiento de la información se realizó en 14 de las 16 aulas que conforman el bloque o cuerpo sur que son ocupadas a diario por los estudiantes que acuden a clase en el Bloque A.

De acuerdo al Servicio de Contratación de Obras (2017), se considera un rango normativo de 1.63 m² por estudiante. El cuerpo sur o bloque A de aulas dispone de una morfología semicircular en la cual se observa la disposición y organización modular de las aulas en hilera en cada planta. Así, en el subsuelo están seis aulas cuyas superficies oscilan entre $49.50 \mathrm{~m}^{2}$ y $76.94 \mathrm{~m}^{2}$, con un promedio de superficie de $65.59 \mathrm{~m}^{2}$, y una capacidad de albergar 40 estudiantes por aula. Al establecer la relación superficie promedio con número de alumnos se obtiene $1.64 \mathrm{~m}^{2} /$ alumno.

La planta baja del cuerpo edificado sur dispone de cinco aulas con superficies entre $74.36 \mathrm{~m}^{2}$ y 96.47 $\mathrm{m}^{2}$, con un promedio de $76.27 \mathrm{~m}^{2}$. De la misma forma si se consideran 40 alumnos promedio por aula, entonces la relación número de alumnos con superficie es igual a $1.91 \mathrm{~m}^{2} /$ alumno. La planta alta presenta cinco aulas con superficies entre $73.66 \mathrm{~m}^{2}$ y $76.94 \mathrm{~m}^{2}$ con un promedio de $76.27 \mathrm{~m}^{2} \mathrm{y}$ una relación superficie por alumno de $1.91 \mathrm{~m}^{2} /$ alumno. Por lo expuesto se evidencia que la relación 
número de alumnos con la superficie cumple con la norma ecuatoriana en las aulas de las tres plantas del edificio.

\section{2. Índice de importancia relativa (IIR)}

El IIR se aplicó para categorizar las variables que son determinantes en el confort ambiental y psicológico por parte de los estudiantes. Fue empleado sobre la base de la escala de Likert cuyos valores estuvieron comprendidos entre 1 (totalmente en desacuerdo) y 5 (totalmente de acuerdo). Con ello, fue factible jerarquizar el orden de las variables que surten mayor impacto en el confort.

IIR varía de 0 a 1 y se calcula como en la ecuación (2), en la que la ponderación dada a cada factor multiplica a los encuestados ( 1 a 5 ) donde 5 es la ponderación más alta por el número total de encuestados. Cuanto mayor sea el IIR, mayor será la clasificación y la importancia percibida del factor que afecta Santamaría \& Valentín (2018).

$$
\mathrm{IIR}=\frac{\sum W}{A \times N}
$$

Al aplicar el índice de importancia relativa IIR fue posible jerarquizar cuál de las veinte variables analizadas tuvo mayor impacto sobre el confort. De estas se trabajó con las diez variables que presentaron mayor IIR. Se llegó a determinar que, en orden decreciente de confort fue el expresado en la tabla 2.

Tabla 2: Factores de Confort e Incomodidad en edificación histórico patrimonial

\begin{tabular}{clc}
\hline RANGO & VARIABLES & IIR \\
\hline 1 & Actividades académicas al interior edificación & \\
2 & histórica & 0.84561 \\
3 & Iluminación natural exterior & 0.84386 \\
4 & Espacios de encuentro y diálogo exterior & 0.81228 \\
5 & Temperatura exterior & 0.80702 \\
6 & Iluminación natural interior & 0.79123 \\
7 & Humedad del aire al interior & 0.71404 \\
8 & Seguridad de espacios interiores & 0.69825 \\
9 & Olores desagradables al interior & 0.67544 \\
10 & Ruido interior & 0.42982 \\
\end{tabular}

Una vez aplicado el IIR, se determinó que no solo existen factores de confort para los estudiantes, sino que también existen factores de incomodidad, tal como se puede evidenciar en la tabla 2 en la cual los valores más altos de IIR corresponden a las variables de confort y los valores de IIR más bajos corresponden a las variables de incomodidad. Entendiéndose de esta forma que las variables más confortables corresponden al rango 1-2-3 en tanto que las variables menos confortables corresponden al rango 8-9-10 de las cuales el rango 10 corresponde a la variable de mayor incomodidad.

Sobre la base de las jerarquías obtenidas a través de IIR se aplicó la prueba de $\mathrm{Chi}^{2}$ de Pearson a esos factores para verificar si estos (de confort como de incomodidad) tienen relación con el confort ambiental, psicológico y las condiciones de habitabilidad en la edificación, frente al sexo y a la edad de los estudiantes. 
De las diez variables estudiadas se consideraron los tres factores de mayor valor de IIR tanto en confort (rangos 1-2-3) como en incomodidad (rangos 8-9-10) para la aplicación de la prueba estadística de $\mathrm{Chi}^{2}$ y verificación de la hipótesis.

Como resultado se obtuvo que, dentro de las variables de mayor valor de confort determinadas, no existe relación entre el confort de las actividades académicas, la iluminación natural exterior ni los espacios de encuentro y diálogo del ambiente exterior con la edad y el sexo de los estudiantes encuestados. Esto se constata debido a que los valores de p en la prueba de $\mathrm{Chi}^{2}$ oscilan entre 0.164 y 0.804 que son valores superiores a 0.05 . Sin embargo, se evidencia que existe una relación muy estrecha entre la variable iluminación natural exterior con el sexo, lo cual se muestra dado que su valor de p es 0.003 menor al establecido que es 0.05 .

Dentro de las variables de mayor valor en lo relacionado a la incomodidad calculada a través de la aplicación del IIR, se determina que no existe relación entre la incomodidad de ruido en el ambiente interior con la edad y el sexo. Así tampoco hay relación entre la incomodidad que genera la presencia de gases $\mathrm{u}$ olores en el ambiente interior versus el sexo y la edad. Esto se corrobora dado que los valores de $p$ en la prueba hipótesis aplicando $\mathrm{Chi}^{2}$ se enmarcan entre 0.515 y 0.894 . Por otra parte, la incomodidad entre el ruido del ambiente exterior y la edad tampoco exhiben relación alguna. No así, la relación de incomodidad que si se evidencia entre el ruido en el ambiente exterior y el sexo lo cual se ve reflejado en el valor de $p$ en la prueba de $\mathrm{Chi}^{2}$ que corresponde a 0.027.

\section{Discusión}

\subsection{Confort ambiental, psicológico y eficiencia energética}

Respecto a la relación de la comodidad de los espacios y funcionalidad frente a los ambientes interior y exterior y a la mayor valoración positiva del ambiente exterior, en lo ambiental se contrapone con lo manifestado por ECCO (2011), quien señala que de octubre a diciembre son los meses lluviosos, por tal razón, el ambiente exterior debería ser menos confortable que el interior. En lo referente al aspecto psicológico, el sentimiento de bienestar expresado por los estudiantes se corrobora con lo expresado por De Garrido (2013), quien manifiesta que el empleo de elementos naturales incide de manera favorable en la salud y el bienestar. Como se expresó anteriormente el edificio se integra al paisaje natural y construido al mantener armonía con los espacios abiertos exteriores, caminerías, circulaciones, jardines con cubre pisos, árboles y arbustos ornamentales que en su conjunto evidencian calidad espacial, formal y estética. Además, el citado autor señala que el empleo de materiales naturales reduce al máximo el estrés. Aspectos que explicarían la razón por la que los estudiantes sienten mayor confort en el exterior que en el interior.

De igual manera en lo que respecta al confort ambiental de los ambientes exterior e interior se observa que existe mayor valoración positiva de aceptación para el ambiente exterior, situación que podría deberse a que según De Garrido (2013), una condición primordial para sentir bienestar es que el ser humano se encuentre formando parte del medio natural.

En relación al confort psicológico al no existir mayor diferencia en el porcentaje de aceptación positiva entre los ambientes interior y exterior, se asumiría que en los dos espacios los estudiantes se sienten confortables, lo cual podría deberse a la confluencia de varios factores como lo señala Fuentes (2002), cuando afirma que el confort psicológico incluye la percepción espacial, visual, auditiva, entre otros. Uno de los aspectos implícitos en la percepción visual es el color. Cabe señalar que las aulas en su interior tienen colores con tonalidades en blanco, y en el exterior de la edificación blanco y rosáceo. Acorde a lo expresado por De Garrido (2013), el color rosa es muy indicado para 
estados de depresión leve y despeja la mente de los pensamientos negativos, en tanto que el color blanco aporta vitalidad y fuerza.

Respecto a la aceptación de la variable de eficiencia energética y sus componentes, existe coincidencia entre los resultados obtenidos de aceptación por los estudiantes con lo manifestado por De Garrido (2013), quien expresa que se precisan de entornos que puedan proveer agua y energía y de este modo garanticen estabilidad, abrigo y sentimientos de bienestar y tranquilidad.

En lo referente a la alta aceptación del confort ambiental interior por parte de las mujeres podría deberse a que la población estudiantil analizada en su mayoría corresponde al sexo femenino y que de acuerdo a lo expresado por Olgyay (2019), las mujeres precisan de un grado centígrado mayor de temperatura que los hombres para lograr su confort térmico interior.

Este confort alcanzado podría ser el resultado de la confluencia de factores formales. El incremento de temperatura en el interior estaría dado en razón que los vanos de ventanas presentan grandes tramos que facilitan el ingreso de la radiación solar a pesar de tener voladizos de protección que por su tamaño no son suficientes para proporcionar sombra. Según lo afirma Hernández, Garmendia, \& Frenández (2012), la radiación solar en las fachadas no contribuye al confort interior de los ambientes por el aumento de temperatura, para suplir esto es preciso que las ventanas tengan elementos de protección solar que proporcionen suficiente sombra.

Otro elemento a considerar es la orientación y emplazamiento del edificio en sentido Sur Norte, factor que facilita la captación de la radiación solar. Del mismo modo Hernández et al. (2012) refiere que el aprovechar la energía solar lleva en ocasiones a extremar aspectos relacionados con la orientación. Igualmente, otro factor que podría incidir en el aumento de la temperatura es la altura de los entrepisos que en este caso debería llevar implícito un mayor o menor volumen de aire al interior de los espacios. Condiciones que, entre otras, redundan en el aumento de temperatura de las aulas y por ello la mayor aceptación por parte del segmento femenino en el $1.33 \%$.

En referencia al segmento etario se debe considerar que la mayoría de estudiantes que valoran de manera positiva al confort ambiental interior, corresponde a los estudiantes más jóvenes. Esto debido a que el mayor porcentaje de la población estudiantil analizada oscila entre los 18 y 20 años. En torno a ello, Olgyay (2019) señala que la edad juega un papel preponderante en el requerimiento de confort. El citado autor manifiesta que son preferencias distintas entre los grupos de personas menores y mayores a 40 años. En el estudio realizado por Galindo (2017), si bien se refiere a un estudio realizado exclusivamente en un ambiente exterior, pese a haber dividido a su grupo de estudio en cuatro segmentos etarios donde se observaron dos grupos sobre los 40 años, no se encontró según lo mencionado, una diferencia significativa en su estudio frente a la preferencia de confort.

\subsection{Espacio físico y normativa}

En relación con la calidad espacial de los ambientes educativos para mantener buenas condiciones de habitabilidad educativa y bienestar personal que apoyen los procesos de enseñanza aprendizaje, se establece un promedio de 40 alumnos por aula. Según lo expresa el Servicio de Contratación de Obras (2017), en el documento Normas Técnicas y Estándares para la construcción de infraestructura educativa, se considera un rango normativo de $1.63 \mathrm{~m}^{2}$ por estudiante. El análisis realizado muestra que los valores obtenidos 1.64 y $1.91 \mathrm{~m}^{2}$ por estudiante superan el valor establecido en la norma lo que implica que el índice de habitabilidad en las aulas garantiza el confort de los estudiantes en su interior. 


\section{3. Índice de importancia relativa}

Las variables que surten mayor incidencia en el confort de los estudiantes según el IIR exhiben los mayores valores en tanto que las variables que generan incomodidad a través del mismo índice se determinan con los valores más bajos. Situación similar se evidencia en el trabajo realizado por Santamaría \& Valentín (2018) que, si bien no está enfocado a la línea del confort, se relacionan con la jerarquización de factores que afectan la fabricación de hormigones. De igual manera Gündüz, Nielsen, \& Özdemir (2013), realizan un estudio referente al ordenamiento de factores de retraso de proyectos de construcción según su importancia en Turquía en el que se emplea el método del IIR, corroborándose que el IIR es una herramienta estadística idónea cuando se requiere jerarquizar variables que emplean encuestas de tipo Likert.

\section{Conclusiones}

La presente investigación fue resuelta en tres fases. La primera corresponde al análisis descriptivo de las percepciones que los estudiantes presentaron sobre el confort ambiental, psicológico y de eficiencia energética. Estudio que se realizó mediante la aplicación de un cuestionario tipo Likert. A ello se sumó el estudio del confort ambiental considerando el sexo y la edad de los estudiantes. La segunda fase está relacionada con el orden jerárquico de las variables ambientales, psicológicas, funcionales y de eficiencia energética que incidieron en el confort de los estudiantes calculado sobre la base del IIR. Finalmente, la tercera fase versa sobre relación de las variables a través de la aplicación de la prueba de $\mathrm{Chi}^{2}$ que además permitió probar la hipótesis y su respectiva pregunta de investigación: ¿Cuál es la percepción sensorial y psicológica que los estudiantes tienen respecto de los parámetros de confort ambiental, psicológico, energético, funcional y condiciones de habitabilidad actuales en el Bloque A de la Facultad de Ciencias Económicas?

Del análisis descriptivo se puede inferir que el mayor porcentaje de aceptación del confort ambiental corresponde al ambiente exterior. Aquí, los estudiantes sienten mayor comodidad en general, de manera específica en las variables: comodidad de espacios, distribución y funcionalidad de los ambientes. Respecto al confort psicológico, la percepción de comodidad es similar tanto para el medio exterior como para el interior, teniendo una mínima ventaja de percepción de comodidad del primero sobre el segundo. En relación a eficiencia energética, pese a tratarse de una edificación histórica, un alto porcentaje de los estudiantes sienten comodidad con la dotación de servicios básicos al momento de ejecutar sus tareas.

De acuerdo a los factores demográficos analizados, edad y sexo, respecto del confort ambiental (temperatura, humedad, asoleamiento, radiación y ruido), se puede concluir que el mayor porcentaje de sensación de confort correspondió al sexo femenino. En lo referente a la edad, es el grupo etario de 18 a 20 años que muestra un mayor porcentaje de confort en el ambiente interior.

En lo que concerniente a la funcionalidad se verificó que la relación estudiante por $\mathrm{m}^{2}$ está dentro del estándar establecido por el Ministerio de Educación. Determinándose que la superficie promedio de las aulas en las tres plantas versus el número promedio de estudiantes que las ocupan cumple con la normativa ecuatoriana vigente en la actualidad. Respecto a las variables de mayor y menor confort se constituyeron las actividades académicas como el factor que brindó mayor confort, en tanto que el ruido exterior fue la variable que generó mayor incomodidad. En la fase final del trabajo estuvo relacionada a la aplicación de la prueba $\mathrm{Chi}^{2}$ misma que permitió evidenciar que las variables 
en su gran mayoría no tienen relación unas con otras salvo el caso de la variable iluminación natural exterior con el sexo, que mostró un alto nivel de relación.

Finalmente, lo citado permite responder a la pregunta formulada poniendo de manifiesto que las condiciones ambientales, psicológicas, funcionales, de eficiencia energética y habitabilidad de esta edificación, que cuenta con valoración histórica y más de seis décadas de antigüedad, cumple con las demandas de confort de los estudiantes. Adicionalmente, se cumplió con el objetivo planteado porque se logró determinar las sensaciones perceptuales que los estudiantes experimentan al desarrollar sus actividades académicas en el bloque "A" de la Facultad de Ciencias Económicas de la UCE. Se logró establecer también la comparación en porcentaje del nivel de aceptación entre el confort ambiental interior con relación al confort ambiental exterior. Además, se compararon los índices de habitabilidad obtenidos en las aulas con el índice establecido en la norma ecuatoriana, cuyos los resultados cumplen lo especificado por la norma.

A futuro es importante que se desarrolle un estudio de monitoreo y medición instrumental de los factores de confort ambiental que permitan en primera instancia realizar una comparación de lo que citan las normativas ambientales para espacios cerrados y posteriormente una confrontación con el presente estudio que muestra las percepciones y sensaciones de los estudiantes.

\section{Contribución de los autores}

En concordancia con la taxonomía establecida internacionalmente para la asignación de créditos a autores de artículos científicos (https://casrai.org/credit/). Los autores declaran sus contribuciones en la siguiente matriz:

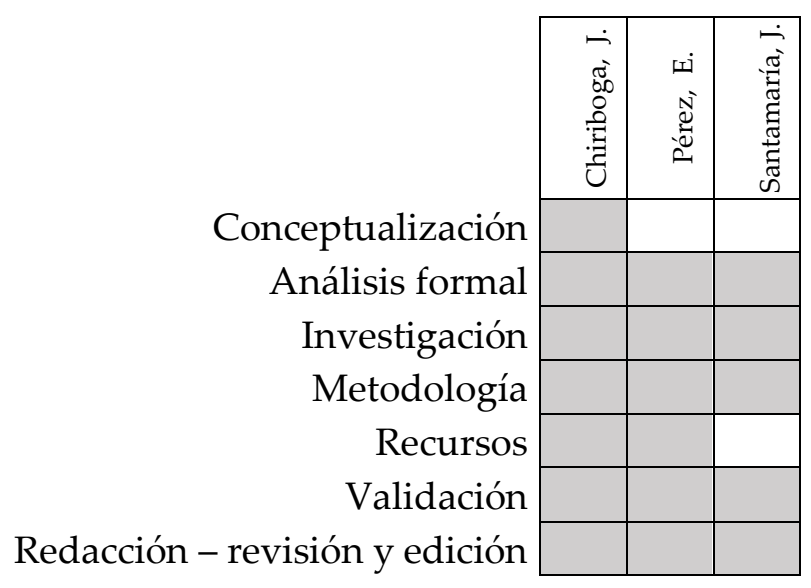

\section{Conflicto de Interés}

En calidad de autores se declara no tener conflictos de interés en ninguna de las etapas de la investigación ni en la publicación del artículo.

\section{Agradecimiento}

Los autores dejan constancia de su agradecimiento a la Comisión de Investigación Formativa de la Dirección de Investigación de la Universidad Central del Ecuador por la ayuda brindada en la asignación de horas, asesoría y fondos económicos otorgados para la ejecución de la presente investigación. 
Además, hacen expreso su agradecimiento al MSc. Byron Francisco Chasi Solórzano, quien orientó parte del manejo estadístico de los datos colectados en el campo.

Finalmente, se hace extensivo el sentimiento de gratitud a la Dra. Nancy Cargua, Coordinadora de la Comisión de Investigación Formativa, por su acertada gestión para viabilizar la ejecución del proyecto. A los arquitectos Luis Bossano y Roberto de la Torre, Coordinadores del Área de Ciencias Exactas, por la asesoría técnica y seguimiento brindados. Así también a la Dra. Nancy Medina, Decana de la Facultad de Ciencias Económicas de la UCE, por haber hecho factible el desarrollo del proyecto en la Facultad de su acertada dirección.

\section{Referencias}

Alías, H. M., Jacobo, G., Martina, P., Corace, J., Aeberhard, R., Coronel, C., Borges, R. \& Yaccuzzi, I. Monitoreo térmico de aulas de la Facultad de Arquitectura de la UNNE (2011) (Resistencia, Chaco) en días de invierno y condiciones reales de ocupación. Avances en Energías Renovables y Medio Ambiente, 15, pp.07.81-07.89 Recuperado de: http://sedici.unlp.edu.ar/handle/10915/101951

Arévalo, J. E. \& Arias, M. A. (2017). Análisis del gradiente térmico para alcanzar condiciones de confort humano, en el aula F1 de la Universidad Politécnica Salesiana. (Trabajo de titulación previo a la obtención del título de Ingeniero Mecánico), Universidad Politécnica Salesiana, Cuenca, Ecuador. https://dspace.ups.edu.ec/bitstream/123456789/14400/1/UPS-CT007071.pdf

Asamblea Nacional del Ecuador. (2019). Registro Oficial año II No. 449. Ley orgánica de Eficiencia Energética. Quito, Ecuador. Recuperado de https://www.recursosyenergia.gob.ec/wpcontent/uploads/downloads/2019/03/Ley-Eficiencia-Energe\%CC\%81tica.pdf

ASHRAE (American Society of Heating, Refrigerating and Air-Conditioning Engineers). (2010). Thermal Environmental Conditions for Human Occupancy. (ANSI/ASHRAE Standard 55) . Atlanta, GA. USA. Recuperado de http://arco-hvac.ir/wp-content/uploads/2015/11/ASHRAE-55-2010.pdf

Baldeón, B. S. (2015). Vivienda bioclimática de interés social para la urbanización "Diosesis de Riobamba" del cantón Guano. Universidad Central del Ecuador, Quito, Ecuador (Trabajo de grado para optar al título de Arquitecto). http://www.dspace.uce.edu.ec/handle/25000/9453

Cardellino, P., Vargas, E. \& Araneda, C. (2017). La evolución del diseño de aula escolar: los casos de Uruguay y Costa Rica. ACE: Architecture, City and Environment, 12 (34), pp. 97-122. Recuperado de https://upcommons.upc.edu/handle/2117/106062

Castejón, J. L. (2006). Escalamiento: Medida de las actitudes, opiniones, sentimientos y percepciones. En A. Alaminos \& J. L. Castejón (Eds.), Elaboración, análisis e interpretación de encuestas, cuestionarios y escalas de opinión (pp. 95-120). Alicante, España: Marfil S.A. Recuperado de https://rua.ua.es/dspace/bitstream/10045/20331/1/Elaboraci\%c3\%b3n,\%20an\%c3\%a1lisis\%2 0e\%20interpretaci\%c3\%b3n.pdf

Cisterna, M., Ledesma, S., Llabra, C., Marquez, S., Martínez, C., Nota, V., Quiñones, G., Mostajo, M., \& Gonzalo, G. (2015). Condiciones de habitabilidad y confort en edificios escolares. Iluminación natural en aulas de escuelas de Tafí del valle, Tucumán. ASADES Energías Renovables y Medio Ambiente. 3, pp.01.25-01.37 Recuperado de: https://bit.ly/3tQTfma

Creative Research System. (1982). Recuperado de https://www.surveysystem.com/sscalc.htm

De Garrido, L. (2013). Arquitectura para la felicidad. Barcelona: Instituto Monsa de Ediciones, S.A. 
ECCO. (2011). Perspectivas del ambiente y cambio climático en el medio urbano. Recuperado de https://biblio.flacsoandes.edu.ec/shared/biblio_view.php?bibid=124087\&tab=opac

Fuentes, V. (2002). Metodología de Diseño Bioclimático: El análisis climático. (Tesis para optar al grado de Maestro en Diseño, Línea Arquitectura Bioclimática), Universidad Autónoma Metropolitana, México. Recuperado de

http://zaloamati.azc.uam.mx/bitstream/handle/11191/5605/Metodologia_diseno_bioclimatic O_Fuentes_2002_MAB.pdf?sequence=1

Galindo, E. T. (2017). El rol moderador de factores individuales y sociales en la percepción térmica exterior de los usuarios de las margenes del rio Tomebamba. (Trabajo de titulación previo a la obtención del título de Magíster en Arquitectura Bioclimática, confort y eficiencia energética), Universidad de Cuenca, Cuenca, Ecuador. Recuperado de http://dspace.ucuenca.edu.ec/handle/123456789/28547

Godoy, F., Palme, M.,Villacreses, G., Beltrán, R. D., Gallardo, A., Almaguer, M., Lobato-Cordero, A. \& Kastillo, J. P. (2017). Estrategias para mejorar las condiciones de habitabilidad y el consumo de energía en viviendas. Instituto Nacional de Eficiencia Energética y Energías Renovables. Recuperado

de https://www.researchgate.net/publication/317239661_Estrategias_para_mejorar_las_condici ones_de_habitabilidad_y_el_consumo_de_energia_en_viviendas_Actualizacion/citation/do wnload

Gómez, L. (2018). Analisis de vulnerabilidad sismica de las instituciones educativas publicas de adobe en el centro histórico de Cajamarca. (Tesis profesional para optar al Título de Ingeniero Civil), Universidad Nacional de Cajamarca, Perú. Recuperado de https://repositorio.unc.edu.pe/handle/UNC/1989

Gündüz, M., Nielsen, Y. \& Ozdemir, M. (2013). Quantification of delay factors using the relative importance index method for construction projects in Turkey. Journal of Management in Engineering, 29(2), pp.133-139. https://ascelibrary.org/doi/10.1061/\%28ASCE\%29ME.19435479.0000129

Hernández, R. J., Garmendia, O. I., \& Frenández-Miranda, M. A. (2012). Arquitectura Ecoeficiente Tomo 1. Recuperado de https://www.academia.edu/32701021/Arquitectura_Ecoeficiente_Tomo_I

INEN. (2009). Eficiencia energética en edificaciones. Requisitos. (Norma Técnica Ecuatoriana NTE INEN 2506:2009). Quito, Ecuador. Recuperado de https://www.normalizacion.gob.ec/buzon/normas/2506.pdf

Jiménez, C., Logroño, M., Rodas, R., \& Yepez, E. (1999). Programa de capacitación en liderzago educativo. Módulo de Tutoría 1. Ecuador: Unidad Técnica EB/PRODEC-MEC-BIRF Ecuador.

Jiménez, L. O. (2017). Estudio de las condicionantes de confort y su aplicación en el rediseño de los espacios interiores del antiguo edificio municipal del cantón Mocha. (Proyecto de investigación previo a la obtención del título de Arquitecto de Interiores), Universidad Técnica de Ambato, Ambato, Ecuador. Recuperado de https://repositorio.uta.edu.ec/jspui/handle/123456789/25715

Ministerio de Desarrollo Urbano y Vivienda. (2018). Eficiencia energética en Edificaciones Residenciales (Norma Ecuatoriana de la Construcción NEC-11) Quito, Ecuador. Recuperado de 
https://www.habitatyvivienda.gob.ec/wp-content/uploads/downloads/2019/03/NEC-HSEE-Final.pdf

Narváez, J. P., Quezada, K. C., \& Villavicencio, R. P. (2015) Criterios bioclimáticos aplicados a los cerramientos verticales y horizontales para la vivienda en Cuenca. (Trabajo de grado previo a la obtención del título de Arquitecto) Universidad de Cuenca, Cuenca, Ecuador. http://dspace.ucuenca.edu.ec/handle/123456789/21783

Olgyay, V. (2019). Arquitectura y Clima: Manual de diseño bioclimático para arquitectos y urbanistas. Barcelona, España: Editorial Gustavo Gili, SL. Recuperado de https://ggili.com/media/catalog/product/9/7/9788425214882_inside.pdf

Ortíz, H. (2016). Bioconstrucción y arquitectura bioclimática para la ejecución de vivienda ecológica unifamiliar. (Tesis de fin de carrera - Grado en Arquitectura Técnica). Universidad Politécnica de Valencia, Valencia, España. http://hdl.handle.net/10251/68553

Peralta, E., \& Moya, R. (2007). Guía Arquitectónica de Quito. Quito, Ecuador: Trama Ediciones.

Riofrío, M. (2019). Análisis del confort térmico de edificaciones construidas con tecnologías de tierra y estructura de madera, en microclimas fríos de la serranía ecuatoriana. (Trabajo de Titulación previo a la obtención del título de Master en Arquitectura y Sostenibilidad). Pontificia Universidad Católica del Ecuador. Quito, Ecuador. http://repositorio.puce.edu.ec/handle/22000/16174

Santamaría, J., \& Valentín, V. (2018). Percepcions on construction-related factors that affect concrete quality, costs and production. Avances en Ciencias e Ingenierias, 10(16), pp.120-139. Recuperado de https://revistas.usfq.edu.ec/index.php/avances/article/view/980/1339

Servicio de Contratación de Obras. (2017). Normas Técnicas y Estándares para la Construcción de Infraestructura Educativa. Memoria Arquitectónica "UEM Pajan" Quito, Ecuador. Recuperado de: https://es.scribd.com/document/431759631/Memoria-Arq-Estandar

Vásquez, G. F. (2017). Análisis del confort térmico dentro de espacios de oficinas en la zona climática 5, caso de estudio edificio del municipio de Tulcán. (Trabajo de titulación previo a la obtención del título de Magister en Arquitectura y Sostenibilidad), Pontificia Universidad Católica del Ecuador, Quito. Ecuador.

http://repositorio.puce.edu.ec/bitstream/handle/22000/15019/ARCHIVO\%20FINAL\%20DE \%20TESIS_12MAYO2018.pdf?sequence=1\&isAllowed=y

Villagómez, J. C., \& Bonilla, X. (2017). La arquitectura de Gilberto Gatto Sobral. En Colegio de Arquitectos del Ecuador Pichincha website: https://www.cae.org.ec/la-arquitectura-degilberto-gatto-sobral/ 